This item was submitted to Loughborough's Research Repository by the author.

Items in Figshare are protected by copyright, with all rights reserved, unless otherwise indicated.

\title{
The mechanics of the backward giant circle on the high bar
}

PLEASE CITE THE PUBLISHED VERSION

\section{PUBLISHER}

YEADON, M.R. and HILEY, M.J., 2000. The mechanics of the backward giant circle on the high bar. Human Movement Science, 19 (2), pp.153-173. (C) Elsevier

VERSION

AM (Accepted Manuscript)

LICENCE

CC BY-NC-ND 4.0

\section{REPOSITORY RECORD}

Yeadon, Maurice R., and Michael J. Hiley. 2019. "The Mechanics of the Backward Giant Circle on the High Bar”. figshare. https://hdl.handle.net/2134/6460. 
This item was submitted to Loughborough's Institutional Repository (https://dspace.lboro.ac.uk/) by the author and is made available under the following Creative Commons Licence conditions.

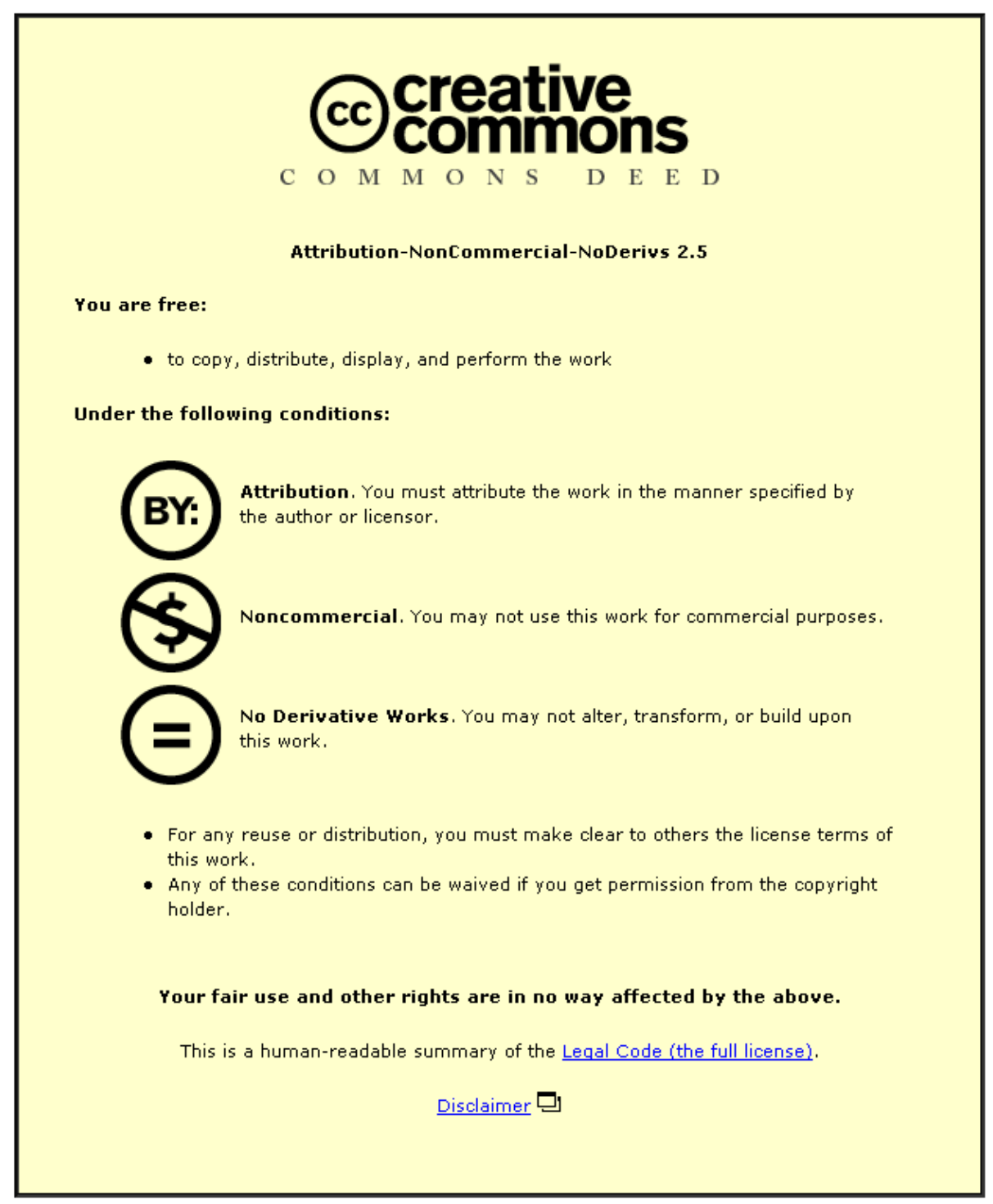

For the full text of this licence, please go to: http://creativecommons.org/licenses/by-nc-nd/2.5/ 


\title{
The mechanics of the backward giant circle on the high bar
}

\author{
Maurice R. Yeadon and Michael J. Hiley \\ Department of Sports Science, Loughborough University, LE11 3TU, UK
}

\begin{abstract}
In Men's Artistic Gymnastics the backward giant circle on the high bar is used to generate the rotation that the gymnast needs to perform the release-regrasp and dismount skills. Bauer (1983) presented a point mass model of high bar circling which indicated that ideally a gymnast should flex around the lowest point and extend around the highest point of a giant circle in order to maximise the increase in energy. In practice gymnasts follow this technique in only a general sense and flex after the lowest point and extend before the highest point. A four segment planar simulation model of a gymnast was developed to investigate these differences in technique. The model comprised arm, torso, thigh and leg segments with a damped linear spring connecting the arm and torso segments. The high bar was also modelled as a damped linear spring. The model was driven using time histories of hip and shoulder angles. It was found that the simplifications introduced into Bauer's model by neglecting segmental inertias and the elastic characteristics of the gymnast and the bar were not responsible for the differences between the ideal technique and the typical technique of gymnasts. The technique differences could be accounted for by limitations on the torques that are exerted at the shoulder and hip.
\end{abstract}

Keywords: biomechanics, high bar, simulation, gymnastics, optimisation

\section{Introduction}

The high bar is one of six pieces of apparatus used in Men's Artistic Gymnastics competitions. A high bar routine consists of a number of circling skills, release-regrasp skills and a dismount. The backward giant circle is used to link the circling skills and to provide the necessary rotation for the release and dismount skills. The basic technique of the backward giant circle comprises a flexion at the hips and shoulders as the gymnast passes through the lower part of the circle and an extension as the gymnast passes through the upper part (Fig. 1).

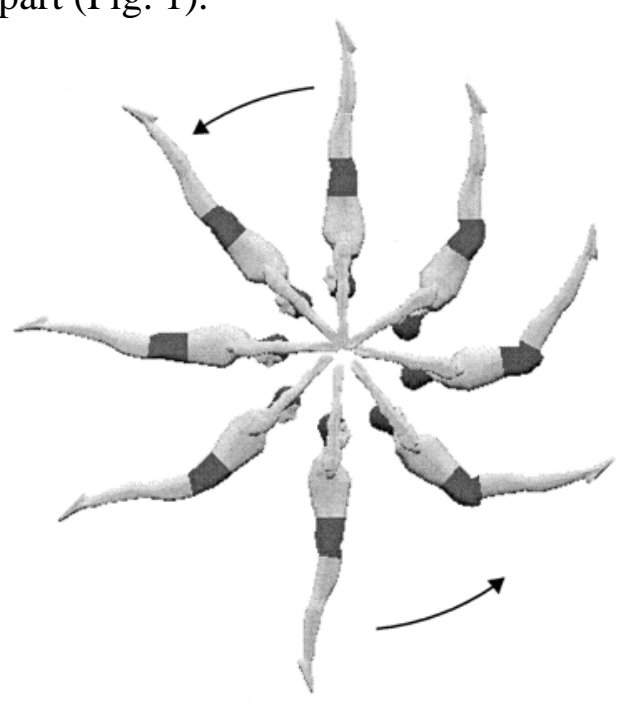

Fig. 1. A backward giant circle. From a handstand position on the high bar the gymnast rotates backwards through $360^{\circ}$. 
Bauer (1983) described the technique of a gymnast performing a backward giant circle using a pendulum of varying length. The mass of the pendulum represented the mass of the gymnast and the length of the pendulum represented the distance from the gymnast's mass centre to the axis of rotation. Using this model Bauer showed that ideally the gymnast should flex instantaneously at the lowest point, when the mass centre of the gymnast is directly beneath the bar, and extend instantaneously at the highest point in order to increase the energy (Fig. 2). The theoretical optimum technique is to flex instantaneously at the lowest point and extend instantaneously at the highest point. Flexing in the lower half of the circle increases the gymnast's potential energy and kinetic energy. Extending through the higher half of the circle increases the gymnast's potential energy whilst the gymnast's kinetic energy decreases only slightly. This explains why gymnasts flex during the lower half of the circle and extend in the higher half of the circle. Since a gymnast will require a finite time to flex it might be expected that the flexion will start a certain time before the lowest point is reached and will end a similar time after passing through the lowest point. Similarly it might be expected that the extension will occur approximately symmetrically around the highest point. However it is clear from the literature that the majority of the flexion occurs after the gymnast has passed through the lowest point of the giant circle (Brüggemann et al., 1994; Cheetham, 1984; Gervais and Tally, 1993; Okamoto et al, 1987). Similarly most gymnasts complete the majority of the extension before the highest point (Okamoto et al., 1987).

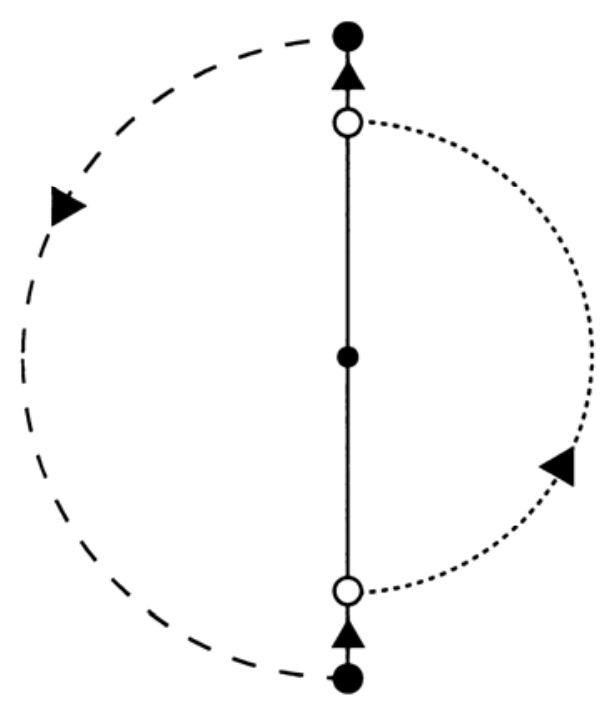

Fig. 2. Giant circle technique suggested by a point mass model (adapted from Bauer, 1983). During the downswing the body is fully extended so that the radius of the semicircle described by the mass centre is as large as possible. At the lowest point the mass centre is raised so that during the upswing the radius is smaller.

During the downswing the radius of the semicircle described by the mass centre is larger than the radius during the upswing. As a consequence the moment of the gymnast's weight about the bar is larger during the downswing than during the upswing. Thus the gain in angular momentum about the bar in the downswing is larger than the decrease in angular momentum in the upswing. The simplified point mass model presented by Bauer (1983) did not take into account the moment of inertia of the gymnast. This aspect may influence the optimum timing of the actions performed by the gymnast. The model also neglected the elastic properties of the bar and the gymnast and again this may influence the phasing of the flexion and extension actions. It is also possible that the phasing of the flexion and extension are related to the strength 
characteristics of the gymnast. It may be that gymnasts are not strong enough to flex around the lowest point or to extend around the highest point.

The purpose of this study was to explain how each of the following aspects influences the timing of the flexion and extension:

- segmental inertias

- elasticity of bar and gymnast

- strength of the gymnast

\section{Method}

A combination of experimental and theoretical approaches was used. Data were collected on an elite gymnast performing backward giant circles. A computer simulation model was personalised for this gymnast and was then used to optimise performance in the backward giant circle.

\section{Data collection}

The subject, a member of the Great Britain Men's Senior Squad, was asked to perform two sets of linked giant circles. The performance was recorded using two genlocked video cameras, a Sony Hi8 Hyper HAD (EVW-300P) and a Sony Digital Handycam (DCR-VX1000E), operating at $50 \mathrm{~Hz}$ with shutter speeds of $1 / 500 \mathrm{~s}$ and $1 / 600 \mathrm{~s}$ respectively. The two cameras were placed $8.5 \mathrm{~m}$ from the high bar at a height of $1.6 \mathrm{~m}$ from the ground (Fig. 3). A photocell was attached to the furthest upright of the high bar from the camera with a reflective disc placed on the nearest upright. The photocell was at such a height that the subject's hips would break the beam each time he passed through the lowest point of the giant circle. The breaking of the photocell beam was used to trigger an LED array in view of both video cameras. This event was used to synchronise the data from the two video cameras.

Prior to the gymnast performing the giant circles a calibration structure comprising 28 spheres of diameter $0.08 \mathrm{~m}$ placed in known locations and spanning a volume measuring $1 \mathrm{~m} \times 5 \mathrm{~m} \times 5 \mathrm{~m}$ was positioned with its centre at the midpoint of the high bar so that it included the volume traced out by the gymnast during a giant circle. The calibration structure was video recorded by both cameras.

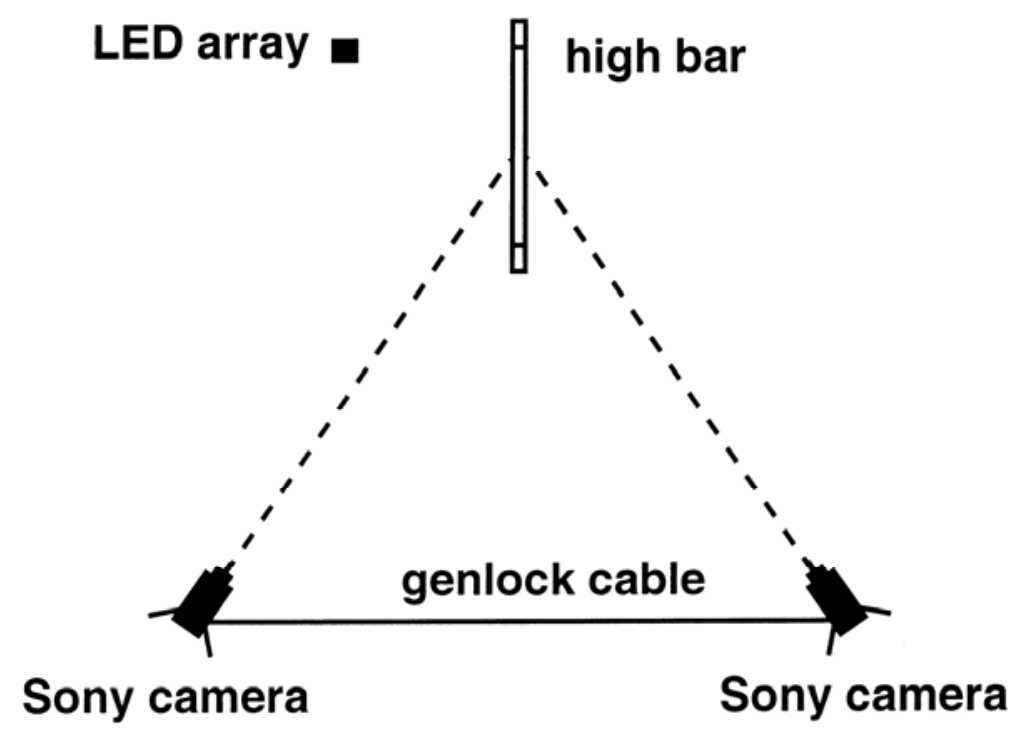

Fig. 3. Location of the cameras relative to the high bar. 


\section{Data processing}

The centres of the calibration spheres were digitised in five video fields from both camera views. All digitising was carried out with the Target high resolution digitising system (Kerwin, 1995). One giant circle from each of the two trials was digitised for each camera view. In each of the movement fields the wrist, elbow, shoulder, hip, knee and ankle joint centres and toes on each side of the body were digitised along with the centre of the subject's head and the centre of the high bar between the subject's hands. Joint centres were assumed to lie on the midlines of adjacent segments when digitising. The data obtained from digitising the images of the calibration spheres together with their known locations were used to calculate the 11 Direct Linear Transformation parameters for each camera (Abdel-Aziz and Karara, 1971). The synchronised digitised coordinate data from each camera view along with the camera parameters were used to reconstruct the three-dimensional locations of the joint centres using the Direct Linear Transformation method. Joint angles for the left and right sides were averaged to produce input for a planar simulation model. Quintic splines (Wood and Jennings, 1979) were used to fit the orientation and joint angle time histories so that derivatives could be obtained (Yeadon, 1990a).

\section{Simulation model}

A four segment planar simulation model was developed comprising arm, torso, thigh and lower leg segments. The high bar and the gymnast's shoulder structure were modelled as damped linear springs (Fig. 4). It was assumed that the mass centre of each segment lay on the line joining the segment joint centres. Input to the simulation model comprised the segmental inertia parameters, the stiffness and damping coefficients of the bar and shoulder springs, the initial displacement and velocity of the bar, the initial angular velocity of the arm, the initial orientation of the arm and the joint angle time histories. The model was driven using joint angle time histories in the form of quintic splines.

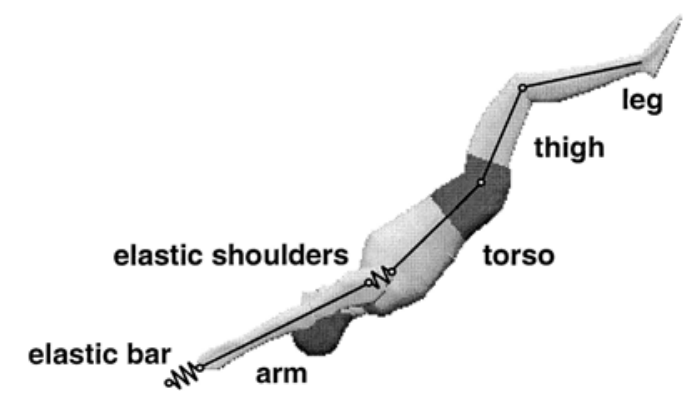

Fig. 4. Four segment simulation model with springs at the bar and shoulder.

The equations of motion were derived using Newton's Second Law of motion and by taking moments about the neutral bar position and the segment mass centres. The system of rigid links and springs was reduced to five equations in five unknowns. The first two equations, (1) and (2), were obtained by resolving the forces horizontally and vertically for the arm segment of the model (Fig. 5). Equations (1) and (2) include the forces exerted by the springs at the bar and shoulder with extensions $x_{b}, z_{b}, x_{s}$ and $z_{s}$ respectively. The stiffness and damping coefficients of the bar and shoulder springs are given by $\mathrm{k}_{\mathrm{bx}}, \mathrm{k}_{\mathrm{bz}}, \mathrm{k}_{\mathrm{s}}, \mathrm{c}_{\mathrm{bx}}, \mathrm{c}_{\mathrm{bz}}$ and $\mathrm{c}_{\mathrm{s}}$.

$$
\begin{gathered}
-\mathrm{k}_{\mathrm{bx}} \mathrm{x}_{\mathrm{b}}-\mathrm{c}_{\mathrm{bx}} \dot{\mathrm{x}}_{\mathrm{b}}+\mathrm{k}_{\mathrm{s}} \mathrm{x}_{\mathrm{s}}+\mathrm{c}_{\mathrm{s}} \dot{\mathrm{x}}_{\mathrm{s}}=\mathrm{m}_{1} \ddot{\mathrm{x}}_{1} \\
-\mathrm{k}_{\mathrm{bz}} \mathrm{z}_{\mathrm{b}}-\mathrm{c}_{\mathrm{bz}} \dot{\mathrm{z}}_{\mathrm{b}}+\mathrm{k}_{\mathrm{s}} \mathrm{z}_{\mathrm{s}}+\mathrm{c}_{\mathrm{s}} \dot{\mathrm{z}}_{\mathrm{s}}-\mathrm{m}_{1} \mathrm{~g}=\mathrm{m}_{1} \ddot{\mathrm{z}}_{1}
\end{gathered}
$$




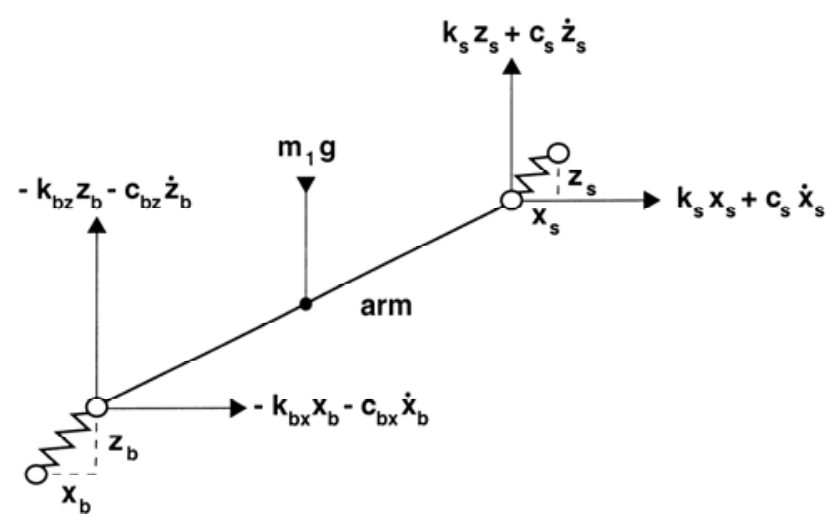

Fig. 5. Free body diagram showing forces acting on the arm segment.

The remaining three equations, (3), (4) and (5), were obtained by resolving forces horizontally and vertically and by taking moments about the neutral bar position $\mathrm{O}$ for the whole model (Fig. 6). The linear accelerations $\ddot{x}_{i}$ and $\ddot{z}_{i}(i=1,4)$, of the four segment mass centres, may be expressed in terms of $\ddot{x}_{b}, \ddot{z}_{b}, \ddot{x}_{s}, \ddot{z}_{s}, \dot{\phi}_{i}$ and $\ddot{\phi}_{i}$. The angular velocities and accelerations at the hip, shoulder and knee joints $\left(\dot{\phi}_{\mathrm{i}}\right.$ and $\left.\ddot{\phi}_{\mathrm{i}}, \mathrm{i}=2,4\right)$ were specified as simulation input (from the joint angle time histories) leading to five equations in five unknowns. The unknowns were the linear accelerations of the springs and the angular acceleration of the arm segment $\left(\ddot{\mathrm{x}}_{\mathrm{b}}, \ddot{\mathrm{z}}_{\mathrm{b}}, \ddot{\mathrm{x}}_{\mathrm{s}}, \ddot{\mathrm{z}}_{\mathrm{s}}\right.$ and $\left.\ddot{\phi}_{1}\right)$. The five linear equations were solved numerically for the five unknowns (Stewart, 1973). A second order Runge-Kutta method was used to advance the solution $\left(\mathrm{x}_{\mathrm{b}}, \mathrm{z}_{\mathrm{b}}, \mathrm{x}_{\mathrm{s}}, \mathrm{z}_{\mathrm{s}}\right.$ and $\left.\phi_{1}\right)$ for a time step of $0.0001 \mathrm{~s}$ calculating also $\dot{\mathrm{x}}_{\mathrm{b}}, \dot{\mathrm{z}}_{\mathrm{b}}, \dot{\mathrm{x}}_{\mathrm{s}}, \dot{\mathrm{z}}_{\mathrm{s}}$ and $\dot{\phi}_{1}$ as part of the numerical integration.

$$
\begin{aligned}
&-\mathrm{k}_{\mathrm{bx}} \mathrm{x}_{\mathrm{b}}-\mathrm{c}_{\mathrm{bx}} \dot{\mathrm{x}}_{\mathrm{b}}=\mathrm{m}_{1} \ddot{\mathrm{x}}_{1}+\mathrm{m}_{2} \ddot{\mathrm{x}}_{2}+\mathrm{m}_{3} \ddot{\mathrm{x}}_{3}+\mathrm{m}_{4} \ddot{\mathrm{x}}_{4} \\
&-\mathrm{k}_{\mathrm{bz}} \mathrm{z}_{\mathrm{b}}-\mathrm{c}_{\mathrm{bz}} \dot{\mathrm{z}}_{\mathrm{b}}-\mathrm{g}\left(\mathrm{m}_{1}+\mathrm{m}_{2}+\mathrm{m}_{3}+\mathrm{m}_{4}\right)=\mathrm{m}_{1} \ddot{\mathrm{z}}_{1}+\mathrm{m}_{2} \ddot{\mathrm{z}}_{2}+\mathrm{m}_{3} \ddot{\mathrm{z}}_{3}+\mathrm{m}_{4} \ddot{\mathrm{z}}_{4} \\
& \text { Torque }=\text { rate of change of angular momentum } \\
&-\mathrm{g}\left(\mathrm{m}_{1} \mathrm{x}_{1}+\mathrm{m}_{2} \mathrm{x}_{2}+\mathrm{m}_{3} \mathrm{x}_{3}+\mathrm{m}_{4} \mathrm{x}_{4}\right)= \mathrm{I}_{1} \ddot{\phi}_{1}+\mathrm{m}_{1} \ddot{\mathrm{z}}_{1} \mathrm{x}_{1}-\mathrm{m}_{1} \ddot{\mathrm{x}}_{1} \mathrm{z}_{1} \\
&+\mathrm{I}_{2} \ddot{\phi}_{2}+\mathrm{m}_{2} \ddot{\mathrm{z}}_{2} \mathrm{x}_{2}-\mathrm{m}_{2} \ddot{\mathrm{x}}_{2} \mathrm{z}_{2} \\
&+\mathrm{I}_{3} \ddot{\phi}_{3}+\mathrm{m}_{3} \ddot{\mathrm{z}}_{3} \mathrm{x}_{3}-\mathrm{m}_{3} \ddot{\mathrm{x}}_{3} \mathrm{z}_{3} \\
&+\mathrm{I}_{4} \ddot{\phi}_{4}+\mathrm{m}_{4} \ddot{\mathrm{z}}_{4} \mathrm{x}_{4}-\mathrm{m}_{4} \ddot{\mathrm{x}}_{4} \mathrm{z}_{4}
\end{aligned}
$$

where $I_{i}$ is the moment of inertia of the $i^{\text {th }}$ segment about its mass centre. 


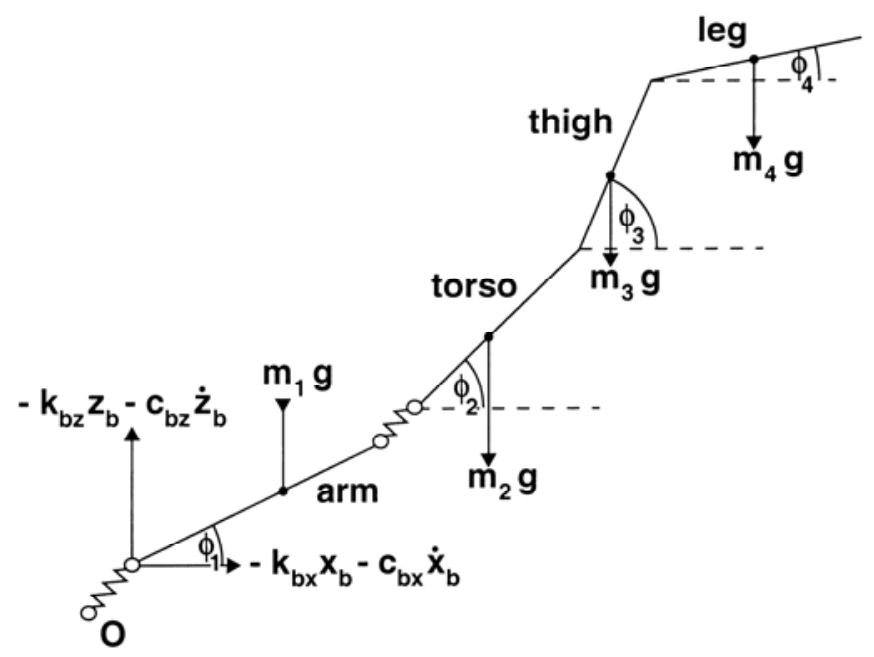

Fig. 6. Free body diagram showing forces acting on the whole body comprising segments 1 (arm), 2 (torso), 3 (thigh) and 4 (leg).

Output from the model comprised the time histories of the horizontal and vertical bar displacements, the rotation angle, the joint torques and the angular momentum of the body about the neutral bar position. The rotation angle was defined as the angle made by the line joining the mass centre of the model to the neutral bar position with the vertical (Fig. 7).

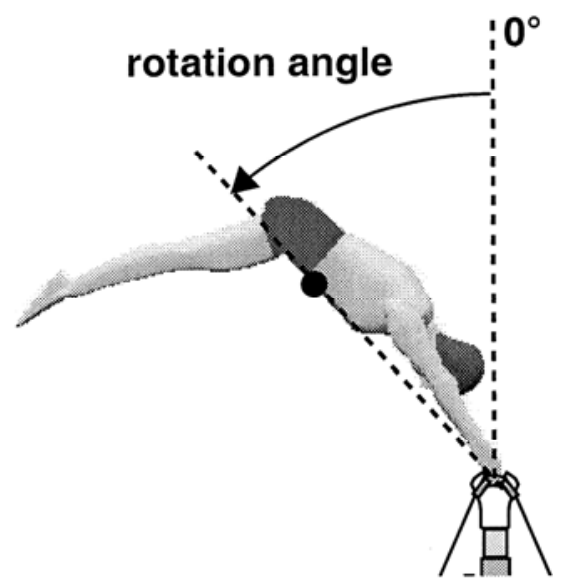

Fig. 7. The rotation angle of the body is the angle between the vertical and a line from the neutral bar position to the mass centre.

The angular momentum of the body about the neutral bar position $\mathrm{O}$ was calculated using equation (6).

$$
\mathrm{h}=\sum_{\mathrm{i}=1}^{4}\left(\mathrm{I}_{\mathrm{i}} \dot{\phi}_{\mathrm{i}}+\mathrm{m}_{\mathrm{i}} \dot{\mathrm{z}}_{\mathrm{i}} \mathrm{x}_{\mathrm{i}}-\mathrm{m}_{\mathrm{i}} \dot{\mathrm{x}}_{\mathrm{i}} \mathrm{z}_{\mathrm{i}}\right)
$$

\section{Parameter determination}

The inertia parameters for the model were obtained from anthropometric measurements of the gymnast who performed the giant circles in the data collection using the inertia model of Yeadon (1990b). The inertia data presented in Table 1 were used in all simulations. Since the model has single segments representing both arms, both thighs and both legs the values given are the combined values for both limbs. The mass centre distance (mcd) for each segment was measured from the joint centre nearer to the bar and the moments of inertia were about the mass centre of each segment. The 
stiffness and damping coefficients for the high bar and shoulder springs were obtained using the four segment simulation model together with the Simulated Annealing optimisation algorithm of Goffe et al. (1994). During the first digitised backward giant circle where the simulation model was driven using splined joint angle time histories, the spring coefficients were manipulated by the Simulated Annealing algorithm so as to minimise the differences between rotation angle and bar displacements and the simulation equivalents. The cost function to be optimised was weighted so that a rms difference of $20^{\circ}$ in the rotation angle was equivalent to a $0.026 \mathrm{~m}$ rms difference in the vertical or horizontal bar displacement. Using Hooke's law a rms difference of $0.026 \mathrm{~m}$ would be equivalent to an error in the estimated reaction force at the bar of approximately one bodyweight.

Table 1. Segmental inertia parameters of the simulation model

\begin{tabular}{|lclll|}
\hline Segment & Mass $(\mathrm{kg})$ & Length $(\mathrm{m})$ & MCD $(\mathrm{m})$ & Moment of inertia $\left(\mathrm{kg} \mathrm{m}^{2}\right)$ \\
\hline Arm & 6.87 & 0.548 & 0.239 & 0.205 \\
\hline Torso & 33.57 & 0.601 & 0.337 & 1.610 \\
\hline Thigh & 14.07 & 0.374 & 0.151 & 0.173 \\
\hline Leg & 7.54 & NA & 0.227 & 0.164 \\
\hline
\end{tabular}

\section{Evaluation of the model}

The simulation was started from a rotation angle of $10^{\circ}$ (just past the handstand position) and finished at a rotation angle of $450^{\circ}$ (when the body was horizontal). The simulation model was driven by the joint angle time histories from the video analysis of the second digitised giant circle. The spring parameter values were those determined from the first digitised giant circle using the optimisation procedure. Comparisons were made between the rotation angles and bar displacements obtained from simulation and video analysis. The root mean squared (rms) differences between the simulation and video values were calculated.

\section{Optimisations}

Four optimisations were performed to investigate why gymnasts flex after passing through the lowest point of a giant circle. The criterion for the optimisations was to maximise the angular momentum of the model gymnast after rotating through an angle of $450^{\circ}$. The Simulated Annealing algorithm was used to manipulate the start and end times of the flexion and extension actions. The simulations performed in each of the optimisations were started with the simulation model fully extended in the handstand position (rotation angle of $0^{\circ}$ ). Each simulation was started with an initial angular velocity so that when passing through a rotation angle of $90^{\circ}$ the model possessed the same angular momentum as the gymnast in the second giant circle from the video analysis. During each simulation the model was required to perform a flexion and extension action at both the hip and shoulder joints. In changing a joint angle $\theta$, from $\theta_{1}$ to $\theta_{2}$ between times $t_{1}$ and $t_{2}$ the time history was given by:

$$
\theta(\mathrm{t})=\theta_{1}+\left(\theta_{2}-\theta_{1}\right) \mathrm{q}(\mathrm{x})
$$

where $\mathrm{x}=\left(\mathrm{t}-\mathrm{t}_{1}\right) /\left(\mathrm{t}_{2}-\mathrm{t}_{1}\right)$ and $\mathrm{q}(\mathrm{x})=\mathrm{x}^{3}\left(6 \mathrm{x}^{2}-15 \mathrm{x}+10\right)$.

Note that $\mathrm{q}(\mathrm{x})$ is a quintic function with the properties $\dot{\mathrm{q}}(0)=\dot{\mathrm{q}}(1)=\ddot{\mathrm{q}}(0)=\ddot{\mathrm{q}}(1)=0$ so that angle changes are effected with zero velocity and acceleration at the endpoints. The flexion action was such that the angles at the hip and shoulder joints were closed by $40^{\circ}$ (Fig. 8). This value corresponded to flexion angles used during the backward giant 
circle in the video analysis. Similarly the extension action corresponded to the angles at the hip and shoulder joints opening by $40^{\circ}$. Three restrictions were placed on the flexion and extension actions. Firstly the flexion had to be performed before the extension. Secondly the angles at the hip and shoulder joints were closed and opened simultaneously by the same amount. Thirdly it was required that the model should also finish the simulation in the fully extended shape: simulations not finishing in the extended shape were given a (angular momentum) score of zero.

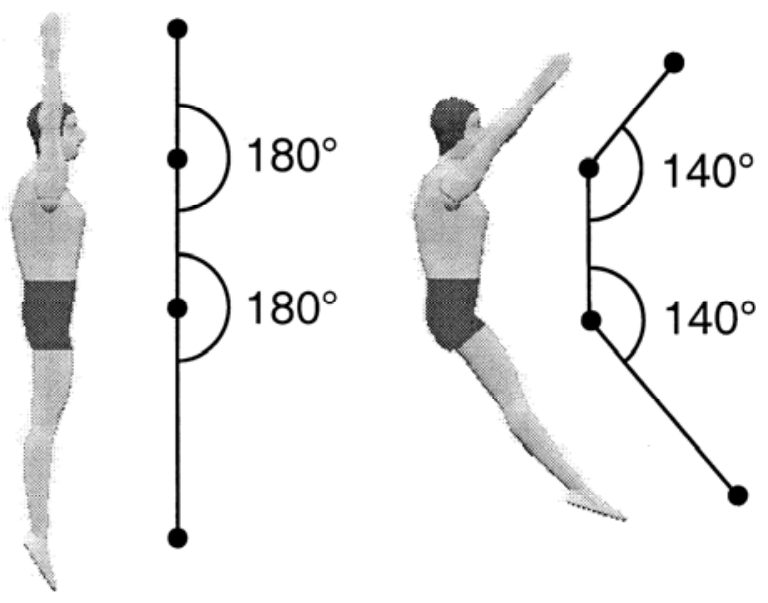

Fig. 8. The angle changes at the hip and shoulder joints used in the simulations.

The separate optimisations were used to address the research question of why gymnasts flex after passing through the lowest point of the giant circle and start to extend before the highest point. In the first optimisation the bar and the gymnast were modelled as inelastic structures in order to determine the effect of segmental inertias on the theoretical optimum technique proposed by Bauer (1983). In the second optimisation the bar and gymnast were modelled as elastic structures in order to determine the effect of the elastic properties of the bar and gymnast on optimum technique. Torque limits at the hip joint and the shoulder joint were used to prevent the displacement of the bar exceeding unrealistic values (approximately $0.20 \mathrm{~m}$ ) but were set high enough $(4000 \mathrm{Nm})$ to comfortably exceed the strength limits of a gymnast. The third optimisation was carried out to determine how the gymnast's strength characteristics influence technique during the backward giant circle. Torque limits at the hip and shoulder joints representative of those of the gymnast were obtained from the simulation based on the video data of the second giant circle. If the torques produced during a simulation exceeded the set limits the simulation was given a (angular momentum) score of zero. The fourth optimisation was a repeat of the third optimisation with the peak shoulder flexion joint torque reduced by $50 \%$.

\section{Results}

\section{Evaluation of the model}

Computer graphics output of the giant circle used in the evaluation is given in Fig. 9. The gymnast maintained an extended configuration during the first quadrant of the giant circle (graphics 1 - 3, Fig. 9), hyper-extended during the second quadrant (graphics 3 5), flexed throughout the third and into the fourth quadrants (graphics 5 - 7) and then extended throughout the remainder of the fourth quadrant and into the beginning of the first quadrant (graphics 7 -10). The angular momentum possessed by the gymnast at 
the end of the giant circle (graphic 11) was $355 \mathrm{~kg} \cdot \mathrm{m}^{2} \cdot \mathrm{s}^{-1}$ corresponding to an angular velocity of 4.54 rad.s ${ }^{-1}$.

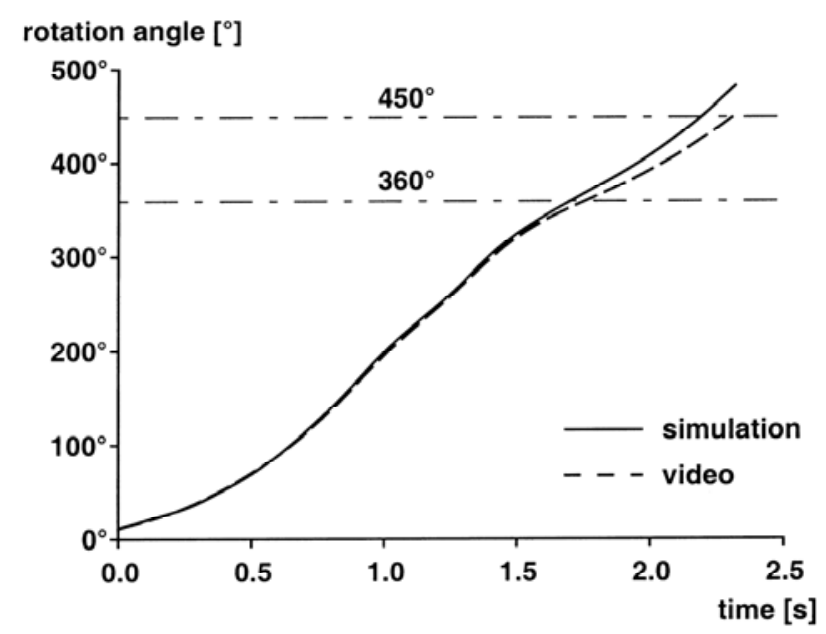

Fig. 9. Graphics sequence of the giant circle used in the video analysis.

A comparison between the rotation angles obtained from video and simulation is given in Fig. 10. The root mean squared difference between the two rotation angle time histories was $9^{\circ}$. This corresponds to an error of $2 \%$ of the total rotation. It can be seen from Fig. 10 that the simulation model closely matches the video data up to a rotation angle of $360^{\circ}$. If the comparison had been made at this point the rms difference would have been less than $3^{\circ}$ which corresponds to a $1 \%$ error.

The rms differences between video and simulation over a rotation of $450^{\circ}$ were $0.009 \mathrm{~m}$ and $0.007 \mathrm{~m}$ for the horizontal and vertical bar displacements respectively. There was a deterioration in the simulated bar displacement after $1.5 \mathrm{~s}$ of the simulation. This was partly due to the errors in the rotation angle. Once the error in rotation angle was sufficiently large the comparisons between the bar displacements obtained from video and simulation were being made at different points of the giant circle. Over the first $360^{\circ}$ of rotation the rms differences in the horizontal and vertical bar displacements were both $0.006 \mathrm{~m}$.

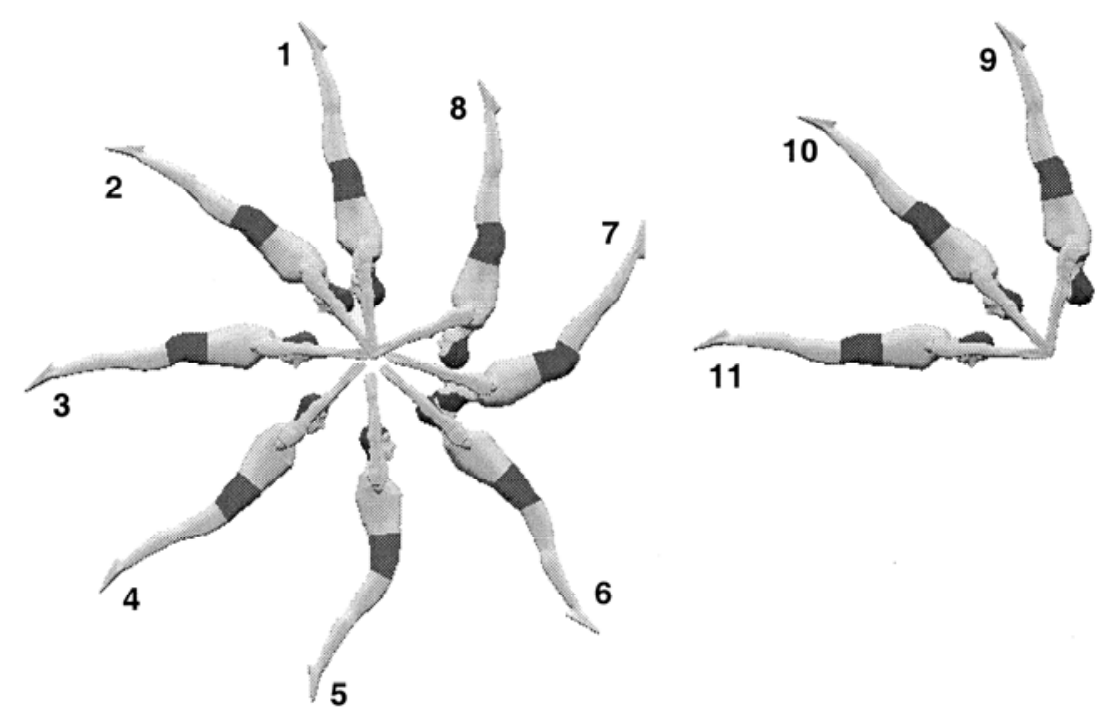

Fig. 10. Comparison between rotation angles obtained from video analysis and simulation. 
The peak internal joint torques obtained during the simulation based on the recorded movement were used to limit the joint torques in the third optimisation. The peak (combined) hip joint torques during the evaluation simulation were $+155 \mathrm{Nm}$ and - $155 \mathrm{Nm}$. These peak joint torques occurred during the flexion and extension actions respectively. The corresponding peak torque values for the (combined) shoulder joint were $+300 \mathrm{Nm}$ and $-155 \mathrm{Nm}$.

\section{Optimisation 1}

To determine the influence of segmental masses and inertias on the optimum timing of the giant circle an optimisation was carried out to maximise the angular momentum with the bar and gymnast modelled as inelastic structures. The angles at which the flexion and extension actions started and ended are shown in Fig. 11. It can be seen that the flexion action is completed before the lowest point and the extension is completed before the highest point (Fig. 11). The final value for the angular momentum about the bar was $401 \mathrm{~kg} \cdot \mathrm{m}^{2} . \mathrm{s}^{-1}$ compared with a value of $316 \mathrm{~kg} \cdot \mathrm{m}^{2} . \mathrm{s}^{-1}$ when the model had passed through $90^{\circ}$ on the first downswing. Therefore, after performing the flexion and extension, the model had increased its angular momentum by $27 \%$.

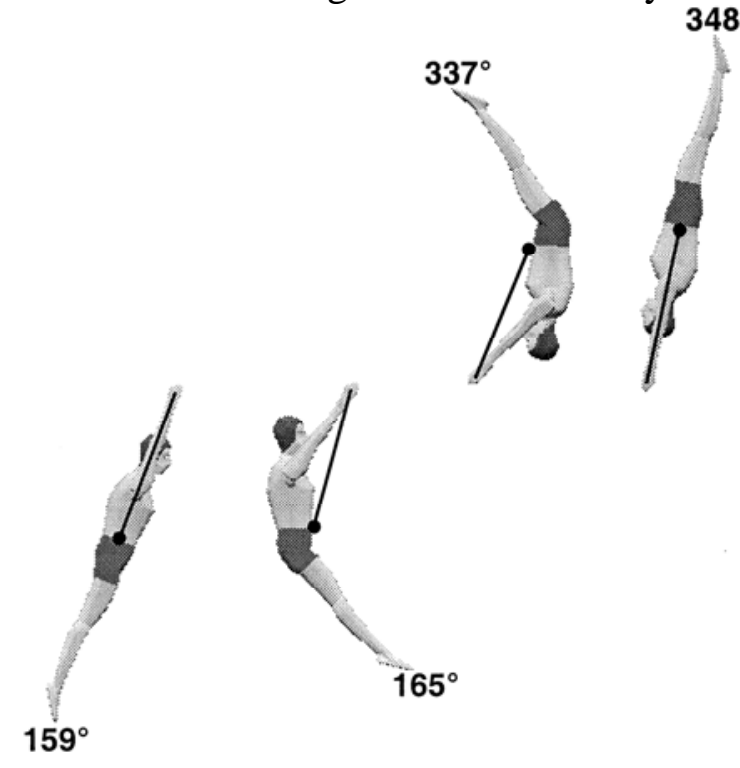

Fig. 11. Optimum technique obtained from the first optimisation with inelastic bar and shoulder joint.

\section{Optimisation 2}

To test whether the result obtained in the first optimisation was influenced by the bar being modelled as an inelastic structure the optimisation procedure was repeated with the bar and gymnast modelled as elastic structures. The final value for the angular momentum about the neutral bar position was $393 \mathrm{~kg} . \mathrm{m}^{2} . \mathrm{s}^{-1}$ which was only marginally less than when the bar and gymnast were modelled as inelastic structures. When compared with the actual performance the angular momentum obtained from the present optimisation was $11 \%$ greater

The angles at which the flexion and extension actions started and ended are given in Fig. 12. When compared with the results from the first optimisation (Fig. 11, inelastic bar and gymnast) there are distinct similarities. The majority of the flexion action is performed before the lowest point and all of the extension action is performed before the highest point. The flexion action in the present optimisation was performed over a larger rotation angle when compared with the first optimisation. This is due in part to 
the $4000 \mathrm{Nm}$ joint torque limit at the hip and shoulder joints introduced to limit the bar displacement.

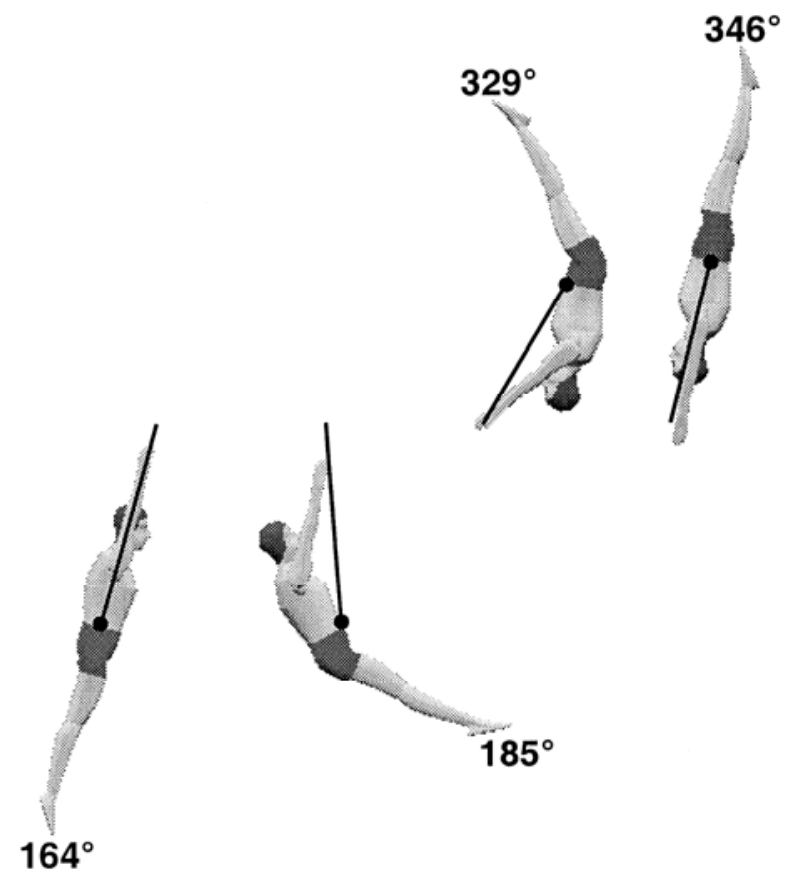

Fig. 12. Optimum technique obtained from the second optimisation with elastic bar and shoulder joint.

The joint torque limit used in the second optimisation was far in excess of a gymnast's strength characteristics. This fact is highlighted by the peak vertical bar displacement which was approximately $0.160 \mathrm{~m}$, some $74 \%$ greater than the value obtained from the video analysis.

\section{Optimisation 3}

Realistic joint torque limits obtained from the simulation based on the video data were introduced during the third optimisation to determine the effect of the gymnast's strength characteristics on the performance of the backward giant circle. As with the second optimisation the bar and gymnast were modelled as elastic structures. The peak vertical displacement of the bar during the optimum solution was $0.096 \mathrm{~m}$ compared with $0.092 \mathrm{~m}$ obtained from the video analysis. The rotation angles at which the flexion and extension actions started and ended are shown in Fig. 13. Comparing Fig. 9 and Fig. 13 it can be seen that the technique used in the optimum solution closely resembles the technique used by a real gymnast. The mid-point of the flexion action, defined as the rotation angle at which the joint angle had completed half the angle change, occurred at a rotation angle of $222^{\circ}$ while the mid-point of the extension action occurred at a rotation angle of $346^{\circ}$. The corresponding mid-points from the video analysis were $225^{\circ}$ and $319^{\circ}$ for the flexion and extension phases respectively. The comparison between the simulated and actual flexion actions is good. In the case of the extension action the real gymnast started the extension action at the hips before the shoulders. The mid-point of the shoulder flexion from the video analysis was $342^{\circ}$. Since during the optimisation the hip and shoulder joints were constrained to flex and extend simultaneously it is likely that the shoulder joint torque limit produced the later midpoint of the extension action when compared with the video analysis. This was found to be the case from the time histories of the joint torques produced by the optimum simulation. 


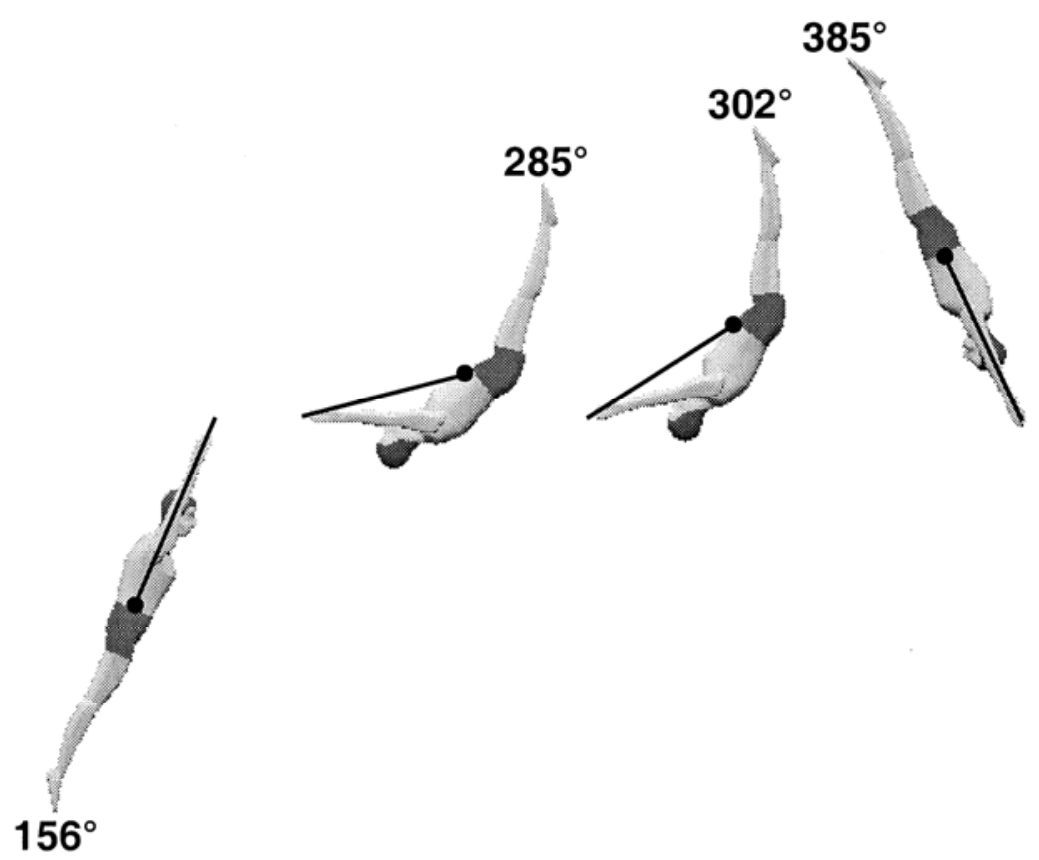

Fig. 13. Optimum technique obtained from the third optimisation with joint torque limits at the hip and shoulder joints.

The final angular momentum for the third optimisation was $366 \mathrm{~kg} \cdot \mathrm{m}^{2} . \mathrm{s}^{-1}$. Compared to the backward giant circle used in the video analysis the optimised technique produced $3 \%$ more angular momentum. The introduction of the realistic joint torque limits reduced the final angular momentum from that obtained from the second optimum by only $7 \%$. Although the torque limits affect the timing of the flexion and extension they do not appear to greatly reduce the final values for the angular momentum generated during the giant circle. This suggests that the gymnast may be limiting the effort used in the flexion.

\section{Optimisation 4}

For the flexion action the joint torque limit at the shoulder was set to $50 \%$ of that in the third optimisation while the hip torque remained unchanged. This may be viewed as either a weaker gymnast or a gymnast using less than maximal effort. As with the second optimisation the bar and gymnast were modelled as elastic structures. The peak vertical displacement of the bar as the model passed through the lowest part of the giant circle was $0.097 \mathrm{~m}$. The rotation angles at which the flexion and extension actions started and ended are given in Fig. 14. Reducing this shoulder joint torque limit resulted in a technique similar to that obtained in the third optimisation with the flexion and extension phases starting and ending later (Fig. 13 and Fig. 14). The mid-point of the flexion action occurred at a rotation angle of $258^{\circ}$ while the mid-point of the extension action occurred at a rotation angle of $353^{\circ}$. The final angular momentum for the fourth optimisation was $341 \mathrm{~kg} \cdot \mathrm{m}^{2} . \mathrm{s}^{-1}$. This is less than the angular momentum possessed by the gymnast in the video analysis. 


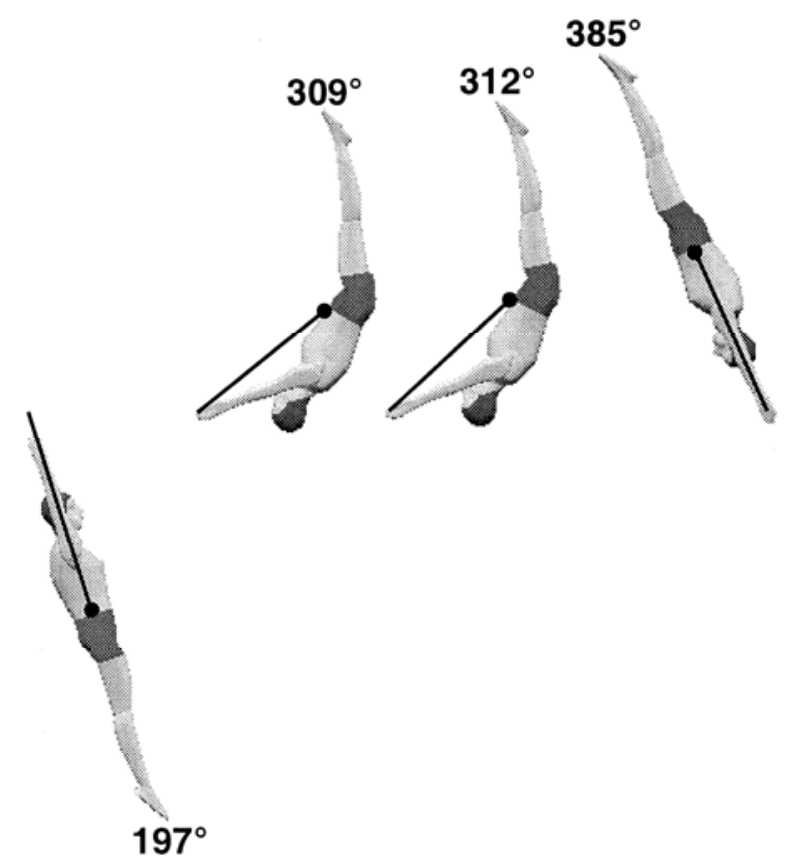

Fig. 14. Optimum technique obtained from the fourth optimisation with shoulder joint torque limit reduced to $50 \%$ of that used in the third optimisation.

\section{Discussion}

The aim of the study was to determine the influence of segmental inertia parameters, bar and gymnast elasticity and the strength characteristics of the gymnast on the technique of the backward giant circle. The answers to these questions were used to explain why gymnasts do not perform the theoretical technique of flexing at the lowest point and extending at the highest point proposed by Bauer (1983).

When the bar and gymnast were modelled as inelastic structures and no limits were placed on the hip and shoulder joint torques the optimum technique for increasing the model's angular momentum about the bar comprised flexion before the lowest point and extension before the highest point (Fig. 11). The reason for the flexion being performed before the lowest point is as follows. During the downswing of a giant circle torques tending to close the hip and shoulder angles must be exerted to maintain the extended configuration (Morlock and Yeadon, 1988; Okamoto et al., 1987). Similarly on the upswing torques tending to open these angles must be exerted to maintain an extended configuration. If at the lowest point the torques at the hip and shoulder joints were set to zero, the model would perform a flexion action (Morlock and Yeadon, 1988). Consider two flexions performed on either side of the lowest point of the giant circle (Fig. 15) with the same change in hip angle and performed over the same duration. The flexion action performed before the lowest point will require a larger torque than the flexion performed after the lowest point and so more concentric work is done and there is a larger increase in energy (e.g. $25 \mathrm{~J}$ more in simulations corresponding to Fig. 15). This is because after the lowest point the model would tend to close the joint angles at the hip and shoulder if the torques at those joints were set to zero and so less work is required to produce the angle change. In order to maximise the increase in energy and the concomitant increase in angular velocity and angular momentum during the flexion, it is of benefit to start the flexion before the lowest point of the giant circle. A similar explanation may be applied to performing the extension before the highest point. If the gymnast passes through the highest point and the joint torques are set to zero the 
gymnast would extend. Larger torques are therefore required to extend before the highest point which in turn leads to more concentric work and a greater increase in energy.

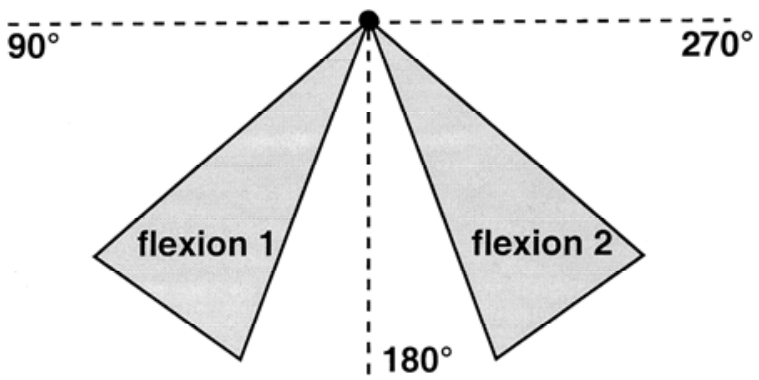

Fig. 15. Two flexion actions performed on either side of the lowest point. Flexion 1 requires a greater joint torque and produces greater angular momentum and energy.

The second optimisation was performed to determine whether the result obtained in the first optimisation was influenced by neglecting the elastic properties of the high bar and gymnast. The elasticity of the bar and gymnast were introduced into the simulation model using damped linear springs. The stiffness and damping coefficients of the springs were obtained by the minimising a cost function which comprised the rms differences between the rotation angles and bar displacements obtained from simulation and video analysis. The solution did not appear to be overly sensitive to the weightings used in the cost function. In the optimised simulation the flexion and extension actions were again performed before both the lowest and highest points of the giant circle, respectively. The mechanical explanation for this result is the same as for the first optimisation.

The third and fourth optimisations were used to determine the influence of the gymnast's strength characteristics on the technique of the backward giant circle. The strength of the model was defined in terms of the joint torques which could be produced at the hip and shoulder joints. The joint torques were limited to maximum values which were not dependent on the joint angular velocity and as such were not intended to model the muscles surrounding the joint. Therefore the joint torque limit may be used to represent either the strength limit of the gymnast or the amount of effort the gymnast uses. Introducing joint torque limits at the hip and shoulder joints produced an optimum technique (Fig. 13) which closely resembled the technique used in the video analysis of the backward giant circle (Fig. 9). The majority of the flexion was performed after the lowest point and the extension was performed passing through the highest point. Comparing the techniques obtained from the second and third optimisations the midpoints of the flexion actions were $176^{\circ}$ and $222^{\circ}$, respectively. In the mechanical analysis of the first and second optimisations it was explained that for two identical flexion actions performed on either side of the lowest point it is the action performed before the lowest point which requires the larger joint torques. It follows then that the introduction of joint torque limits forces the flexion to be performed later. The same can be said of the extension through the upper part of the giant circle where larger torques are required to extend before the highest point. As a consequence the extension in the third optimisation occurs at a later point of the circle than in the other two optimisations. Thus the fact that gymnasts perform the majority of the flexion after the lowest point and the extension through the highest point of a giant circle can be explained by the maximum joint torques being limited. This may be a consequence of the limits of the gymnast's strength or it may be related to the maximum amount of 
effort which the gymnast chooses to use. In the fourth optimisation, when the strength of the model was reduced, the flexion and extension actions were performed later than in the third optimisation as would be expected from the previous explanation.

\section{References}

Abdel-Aziz, Y.I., and Karara, H.M. (1971). Direct linear transformation from comparator coordinates into object space coordinates in close-range photogrammetrey (ASP Symposium on Close-Range Photogrammetry). Falls Church, VA: American Society of Photogrammetry. pp. 1-18.

Bauer, W.L. (1983). Swinging as a way of increasing the mechanical energy in gymnastic maneuvers. In H. Matsui and K. Kobayashi (Eds.), Biomechanics VIIIB. Champaign, IL: Human Kinetics. pp. 801-806.

Brüggemann, G-P., Cheetham, P.J., Alp, Y. and Arampatzis, D. (1994). Approach to a biomechanical profile of dismounts and release-regrasp skills of the high bar. Journal of Applied Biomechanics, 10, 291-312.

Cheetham, P.J. (1984). Horizontal bar giant swing centre of gravity motion comparisons. In J. Terauds, K. Bartheis, R. Mann and J. Crakes (Eds.), Proceedings: International Symposium of Biomechanics in Sports. Colorado Springs: ISBS. pp. 99-108.

Gervais, P. and Tally, F. (1993). The beat swing and mechanical descriptors of three horizontal bar release-regrasp skills. Journal of Applied Biomechanics, 9, 66-83.

Goffe, W.L., Ferrier, G.D. and Rogers, J. (1994). Global optimisation of statistical functions with simulated annealing. Journal of Econometrics, 60, 65-99.

Kerwin, D.G. (1995). Apex/Target high resolution video digitising system. In Proceedings of the Biomechanics Section of the British Association of Sports and Exercise Science. Glasgow: BASES. pp. 1-4.

Morlock, M.M. and Yeadon, M.R. (1988). A mechanical model of a gymnast at the high bar. In C.E. Cotton, M. Lamontagne, D.G.E. Robinson and J.P. Stolhart (Eds.), Proceedings of the Canadian Society for Biomechanics. London, Ont: Spodym. pp. 176-177.

Okamoto, A., Sakurai, S., Ikegami, Y. and Yabe, K. (1987). Mechanical work in the giant swing on the horizontal bar. In G. de Groot, A.P. Hollander, P.A. Huijing and G.J. van Ingen Schenau (Eds.), Biomechanics XI-B. Amsterdam: Free University Press. pp. 758-762

Stewart, G.W. (1973). Introduction to matrix computations. London: Academic Press.

Wood, G.A. and Jennings, L.S. (1979). On the use of spline functions for data smoothing. Journal of Biomechanics, 12, 477-479.

Yeadon, M.R. (1990a). The simulation of aerial movement-I. The determination of orientation angles from film data. Journal of Biomechanics, 23, 59-66.

Yeadon, M.R. (1990b). The simulation of aerial movement-II. A mathematical inertia model of the human body. Journal of Biomechanics, 23, 67-74. 